\title{
Anisotropic complex structure on the pseudo-Euclidean Hurwitz pairs
}

\author{
by W. Królikowski (Eódź)
}

\begin{abstract}
The concept of supercomplex structure is introduced in the pseudoEuclidean Hurwitz pairs and its basic algebraic and geometric properties are described, e.g. a necessary and sufficient condition for the existence of such a structure is found.
\end{abstract}

1. Introduction. In $1923 \mathrm{~A}$. Hurwitz [2] proved that any normed division algebra over $\mathbb{R}$ with unity is isomorphic to either $\mathbb{R}, \mathbb{C}, \mathbb{H}$ or $\mathbb{O}$, the real, complex, quaternion or octonion number algebras. In particular, Hurwitz showed that all the positive integers $n$ and all the systems $c_{j \alpha}^{k} \in \mathbb{R}$, $j, k, \alpha=1, \ldots, n$, such that the collection of bilinear forms $\eta_{j}:=x_{\alpha} c_{j \alpha}^{k} y_{k}$ satisfies the condition

$$
\sum_{j} \eta_{j}^{2}=\left(\sum_{\alpha} x_{\alpha}^{2}\right)\left(\sum_{k} y_{k}^{2}\right)
$$

are restricted to the cases $n=1,2,4$ or 8 .

The results of Hurwitz were the starting point for Ławrynowicz and Rembieliński to introduce the concept of the so-called Hurwitz pairs. They developed the theory obtaining many interesting results. Using the geometric concept of pseudo-Euclidean Hurwitz pairs, they gave their systematic classification in connection with real Clifford algebras. Moreover, they showed that the theory of Hurwitz pairs provided a convenient framework for some problems in mathematical physics (e.g. Dirac equation, Kałuża-Klein theories, spontaneous symmetry breaking and others).

We generalize the concept of supercomplex structure introduced by Lawrynowicz and Rembieliński [3] to pseudo-Euclidean Hurwitz pairs. We describe the basic algebraic and geometric properties of supercomplex structures and find a necessary and sufficient condition for their existence. This is the main result of our paper. We prove that if $O(n, k)$ denotes the orthogonal group preserving the norm $x_{1}^{2}+\ldots+x_{n}^{2}-x_{n+1}^{2}-\ldots-x_{n+k}^{2}$ then 
a complex structure $J\left(J \in O(n, k), J^{2}=-I_{n+k}\right.$, where $I_{n+k}$ stands for the identity $(n+k) \times(n+k)$-matrix) exists if and only if $n$ and $k$ are even.

The concept of a supercomplex structure for Hurwitz pairs is strongly motivated by possible quantum-mechanical applications of anisotropic Hilbert spaces (see e.g. [5]).

2. Pseudo-Euclidean Hurwitz pairs and Clifford algebras. Let us recall fundamental notions and basic results from the theory of pseudoEuclidean Hurwitz pairs. More details can be found in [3-5].

Consider two real vector spaces $S$ and $V$, equipped with non-degenerate pseudo-Euclidean real scalar products $(,)_{S}$ and $(,)_{V}$ with standard properties (see e.g. [3]). For $f, g, h \in V, a, b, c \in S$ and $\alpha, \beta \in \mathbb{R}$ we assume that

$$
\begin{aligned}
& (a, b)_{S} \in \mathbb{R}, \quad(f, g)_{V} \in \mathbb{R}, \\
& \begin{array}{ll}
(b, a)_{S}=(a, b)_{S}, & (g, f)_{V}=\delta(f, g)_{V}, \quad \delta=1 \text { or }-1, \\
(\alpha a, b)_{S}=\alpha(a, b)_{S}, & (\alpha f, g)_{V}=\alpha(f, g)_{V},
\end{array} \\
& (a, b+c)_{S}=(a, b)_{S}+(a, c)_{S}, \quad(f, g+h)_{V}=(f, g)_{V}+(f, h)_{V} .
\end{aligned}
$$

In $S$ and $V$ we choose some bases $\left(\varepsilon_{\alpha}\right)$ and $\left(e_{j}\right)$, respectively, with $\alpha=$ $1, \ldots, \operatorname{dim} S=p ; j=1, \ldots, \operatorname{dim} V=n$. We assume that $p \leq n$. Set

$$
\eta \equiv\left[\eta_{\alpha \beta}\right]:=\left[\left(\varepsilon_{\alpha}, \varepsilon_{\beta}\right)_{S}\right], \quad \kappa \equiv\left[\kappa_{j k}\right]:=\left[\left(e_{j}, e_{k}\right)_{V}\right] .
$$

By (1), we immediately get

$$
\begin{aligned}
\operatorname{det} \eta \neq 0, & \eta^{-1} \equiv\left[\eta^{\alpha \beta}\right], & \eta^{T}=\eta, \\
\operatorname{det} \kappa \neq 0, & \kappa^{-1} \equiv\left[\kappa^{j k}\right], & \kappa^{T}=\delta \kappa .
\end{aligned}
$$

Now, without any loss of generality, we can choose the bases $\left(\varepsilon_{\alpha}\right)$ in $S$ and $\left(e_{j}\right)$ in $V$ so that

$$
\begin{array}{ll}
\eta=\operatorname{diag}(\underbrace{1, \ldots, 1}_{r}, \underbrace{-1, \ldots,-1}_{s}), \quad r+s=p, \\
\kappa=\operatorname{diag}(\underbrace{1, \ldots, 1}_{k}, \underbrace{-1, \ldots,-1}_{l}), \quad k+l=n,
\end{array}
$$

and hence $\eta^{-1}=\eta, \kappa^{-1}=\kappa$.

Next, multiplication of elements of $S$ by elements of $V$ is defined as a mapping $F: S \times V \rightarrow V$ with the properties

(i) $F(a+b, f)=F(a, f)+F(b, f)$ and $F(a, f+g)=F(a, f)+F(a, g)$ for $f, g \in V$ and $a, b \in S$, tion,

(ii) $(a, a)_{S}(f, g)_{V}=(F(a, f), F(a, g))_{V}$, the generalized Hurwitz condi- 
(iii) there exists a unit element $\varepsilon_{0}$ in $S$ for multiplication; $F\left(\varepsilon_{0}, f\right)$ $=f$ for $f \in V$.

The product $a \cdot f:=F(a, f)$ is uniquely determined by the multiplication scheme for base vectors:

$$
F\left(\varepsilon_{\alpha}, e_{j}\right)=C_{j \alpha}^{k} e_{k}, \quad \alpha=1, \ldots, p ; j, k=1, \ldots, n .
$$

Hereafter we shall require the irreducibility of the multiplication $F$ : $S \times V \rightarrow V$, which means that it does not leave invariant proper subspaces of $V$. In such a case we shall call $(V, S)$ a pseudo-Euclidean Hurwitz pair.

It turns out that the generalized Hurwitz condition is equivalent to the relations

$$
C_{\alpha} C_{\beta}^{+}+C_{\beta} C_{\alpha}^{+}=2 \eta_{\alpha \beta} I_{n}, \quad \alpha, \beta=1, \ldots, p,
$$

where we use matrix notation

$$
C_{\alpha}:=\left[C_{j \alpha}^{k}\right], \quad C_{\alpha}^{+}:=\kappa C_{\alpha}^{T} \kappa^{-1},
$$

and $I_{n}$ stands for the identity $n \times n$-matrix. On setting

$$
C_{\alpha}=i \gamma_{\alpha} C_{t}, \quad t \text { fixed, } \alpha=1, \ldots, p, \alpha \neq t,
$$

where $i$ denotes the imaginary unit, we arrive at the following system equivalent to $(5)$ :

$$
\left\{\begin{array}{l}
C_{t} C_{t}^{+}=\eta_{t t} I_{n}, \quad t \text { fixed, } \\
\gamma_{\alpha}^{+}=-\gamma_{\alpha}, \quad \operatorname{Re} \gamma_{\alpha}=0, \alpha=1, \ldots, p, \alpha \neq t \\
\gamma_{\alpha} \gamma_{\beta}+\gamma_{\beta} \gamma_{\alpha}=2 \widehat{\eta}_{\alpha \beta} I_{n}, \quad \alpha, \beta=1, \ldots, p, \alpha, \beta \neq t
\end{array}\right.
$$

where

$$
\widehat{\eta}_{\alpha \beta}:=\eta_{\alpha \beta} / \eta_{t t}
$$

$\left[\eta_{\alpha \beta}\right]$ is the matrix $(3)$. Clearly $\eta_{t t}=1$ or -1 .

From (8) it follows that $\left\{\gamma_{\alpha}\right\}$ are generators of a real Clifford algebra $C^{(r, s-1)}$ or $C^{(r-1, s)}$ with $(r, s-1)$ and $(r-1, s)$ determined by the signature of $\widehat{\eta}:=\left[\widehat{\eta}_{\alpha \beta}\right]$ and by $r+s=p$. Thus, following Ławrynowicz and Rembieliński [3] we have

THEOREM 1. The problem of classifying pseudo-Euclidean Hurwitz pairs $(V, S)$ is equivalent to the classification problem for real Clifford algebras $C^{(r, s)}$ with generators $\left\{\gamma_{\alpha}\right\}$ imaginary and antisymmetric or symmetric according as $\alpha \leq r$ or $\alpha>r$, given by the formulae

$$
\begin{aligned}
& i \gamma_{\alpha} C_{t}=C_{\alpha}, \quad \alpha=1, \ldots, r+s, \quad \alpha \neq t, \\
& C_{t} C_{t}^{+}=\eta_{t t} I_{n}, \quad t \text { fixed },
\end{aligned}
$$

the matrices $C_{\alpha}$ being determined by (2), (5) and (6). The relationship is given by the formulae (8). 
Corollary 1. Without any loss of generality, in Theorem 1 we may set $C_{t}=I_{n}$ and $t=r$, so that $\eta_{t t}=1$ and $\widehat{\eta}_{\alpha \beta}=\eta_{\alpha \beta}$ for $\alpha, \beta \neq t$.

Lemma 1. Pseudo-Euclidean Hurwitz pairs are of bidimension $(n, p)$, $n=\operatorname{dim} V, p=\operatorname{dim} S=r^{\prime}+s^{\prime}+1$,

$$
n= \begin{cases}2^{[p / 2-1 / 2]} & \text { for } r^{\prime}-s^{\prime} \equiv 6,7,0(\bmod 8), \\ 2^{[p / 2+1 / 2]} & \text { for } r^{\prime}-s^{\prime} \equiv 1,2,3,4,5(\bmod 8),\end{cases}
$$

where [] stands for the function "entier".

3. Supercomplex structure: an anisotropic complex structure involving a real Clifford algebra connected with the pseudoEuclidean Hurwitz pairs

Definition. A Hurwitz type vector space $\boldsymbol{E}$ on $(V, \kappa)$ is the $p$-dimensional subspace of the space $\operatorname{End}(V, \kappa)(\operatorname{dim} \operatorname{End} V=\operatorname{dim} V)$ of endomorphisms of $(V, \kappa)$, which consists of all endomorphisms $E$ not leaving invariant proper subspaces of $V$, with the property

$$
(E f, E f)_{V}=\|E\|^{2}(f, f)_{V} \quad \text { for } f \in V, E \in \boldsymbol{E},
$$

where $\|E\|:=\left(\operatorname{Tr} E^{T} E\right)^{1 / 2}, E^{T} E$ being considered in an arbitrary matrix representation of $E$ in an orthonormal basis $\left(e_{j}\right)$ of $V$. We assume that $\boldsymbol{E}$ contains the identity endomorphism $E_{0}$.

Consider next a system $\left(\gamma_{\alpha}\right)$ of $p-1$ imaginary $n \times n$ matrices determined by the formulae

$$
\begin{gathered}
\gamma_{\alpha} \gamma_{\beta}+\gamma_{\beta} \gamma_{\alpha}=2 \widehat{\eta}_{\alpha \beta} I_{n}, \quad \alpha, \beta=1, \ldots, p, \alpha, \beta \neq t \\
\gamma_{\alpha}^{+}=-\gamma_{\alpha}, \quad \operatorname{Re} \gamma_{\alpha}=0, \alpha=1, \ldots, p, \alpha \neq t, \\
\gamma_{\alpha}^{+}:=\kappa \gamma_{\alpha}^{T} \kappa^{-1},
\end{gathered}
$$

where $I_{n}$ is the identity $n \times n$-matrix and $\widehat{\eta}_{\alpha \beta}$ is determined by (9). Then the matrices $\gamma_{\alpha}$ generate a real Clifford algebra. Choose the basic endomorphism $\left(E_{0}, E_{\alpha}\right), \alpha=1, \ldots, p, \alpha \neq t$ in $\boldsymbol{E}$ so that

(11) $E_{0} e_{j}=e_{j}, \quad E_{\alpha} e_{j}=i \gamma_{j \alpha}^{k} e_{k}, \quad \alpha=1, \ldots, p, \alpha \neq t, j, k=1, \ldots, n$,

where $i$ denotes the imaginary unit. The choice (11) is motivated by

LEMma 2. The endomorphisms $E_{0}, E_{\alpha}$ satisfy the relations

(12) $E_{0}=E_{I}, \quad E_{\alpha} e_{j}=C_{j \alpha}^{k} e_{k}, \quad E_{I}$ the identity endomorphism in $\boldsymbol{E}$,

for $\alpha=1, \ldots, p, \alpha \neq t, j, k=1, \ldots, n$, where $C_{j \alpha}^{k}$ can be chosen as

$$
C_{\alpha}=i \gamma_{\alpha}, \quad \alpha=1, \ldots, p, \alpha \neq t, C_{t}=I_{n} .
$$

Proof. The lemma follows directly from (8) and Corollary 1. 
Consider a fixed direction in $\boldsymbol{E}$ determined by the endomorphisms $E_{\alpha}$, $\alpha=1, \ldots, p, \alpha \neq t$. Define

$$
\widetilde{n}:=\sum_{\substack{\alpha=1 \\ \alpha \neq t}}^{p} E_{\alpha} n^{\alpha}, \quad \sum_{\substack{\alpha, \beta=1 \\ \alpha, \beta \neq t}}^{p} \widehat{\eta}_{\alpha \beta} n^{\alpha} n^{\beta}=1
$$

where $\left(n^{\alpha}\right)$ is a system of $p-1$ real numbers. Then we have

LEMMA 3. The endomorphisms $E_{0}$ and $\widetilde{n}$ replace 1 and $i$ of $\mathbb{C}$ in the field of "numbers" $q E_{0}+s \tilde{n}$, where $q, s \in \mathbb{R}$ :

$$
E^{2}=E_{0}, \quad E_{0} \widetilde{n}=\widetilde{n} E_{0}=\widetilde{n}, \quad \widetilde{n}^{2}=-E_{0} .
$$

Pr oof. We only prove the third equality. Notice that

$$
\begin{aligned}
\widetilde{n}^{2}\left(e_{j}\right) & =\widetilde{n}\left(\widetilde{n} e_{j}\right)=E_{\beta} n^{\beta}\left(E_{\alpha} n^{\alpha}\right) e_{j} \\
& =-n^{\alpha} n^{\beta} \gamma_{j \alpha}^{k} \gamma_{k \beta}^{m} e_{m}=-n^{\alpha} n^{\beta}\left[\gamma_{\alpha} \gamma_{\beta}\right]_{j}^{m} e_{m} .
\end{aligned}
$$

On the other hand, we have

$$
\widetilde{n}^{2}\left(e_{j}\right)=-n^{\beta} n^{\alpha}\left[\gamma_{\beta} \gamma_{\alpha}\right]_{j}^{m} e_{m}
$$

Using the above equalities we obtain

$$
\begin{aligned}
2 \widetilde{n}^{2}\left(e_{j}\right) & =-n^{\alpha} n^{\beta}\left[\gamma_{\alpha} \gamma_{\beta}+\gamma_{\beta} \gamma_{\alpha}\right]_{j}^{m} e_{m}=-2 n^{\alpha} n^{\beta} \widehat{\eta}_{\alpha \beta}\left[I_{n}\right]_{j}^{m} e_{m} \\
& =-2\left(n^{\alpha} n^{\beta} \widehat{\eta}_{\alpha \beta}\right) \delta_{j}^{m} e_{m}=-2 e_{j}=-2 E_{0}\left(e_{j}\right) .
\end{aligned}
$$

Hence $\widetilde{n}^{2}=-E_{0}$, as required.

The endomorphism $\widetilde{n}$ is represented in the basis $\left(e_{j}\right)$ by the matrix

$$
J=i n^{\alpha} \gamma_{\alpha} .
$$

Now, we shall show some important properties of this matrix.

REMARK 1. $J^{2}=-I_{n}$.

Pr o of. On the one hand, by the definition we have

$$
J^{2}=\left(i n^{\alpha} \gamma_{\alpha}\right)\left(i n^{\beta} \gamma_{\beta}\right)=-n^{\alpha} n^{\beta} \gamma_{\alpha} \gamma_{\beta} .
$$

On the other hand, changing the indices we get $J^{2}=-n^{\beta} n^{\alpha} \gamma_{\beta} \gamma_{\alpha}$. Thus,

$$
2 J^{2}=-n^{\alpha} n^{\beta}\left[\gamma_{\alpha} \gamma_{\beta}+\gamma_{\beta} \gamma_{\alpha}\right]=-2 n^{\alpha} n^{\beta} \widetilde{\eta}_{\alpha \beta} I_{n}=-2 I_{n} .
$$

Denote by $O(k, l)$ the group of orthogonal transformations of the space $(V, \kappa)(\kappa=\operatorname{diag}(\underbrace{1, \ldots, 1}_{k}, \underbrace{-1, \ldots,-1}_{l}))$. It is well-known that a matrix $B$ belongs to $O(k, l)$ if and only if

$$
B^{T} \kappa B=\kappa \quad \text { or } \quad B \kappa B^{T}=\kappa .
$$


By the definition of the conjugation "+", given in (6), the above condition is equivalent to

$$
B^{+} B=I_{n} \quad \text { or } \quad B B^{+}=I_{n} .
$$

REMARK 2. $J \in O(k, l)$.

Proof. Directly by the definition of $J$ we have

$$
J \kappa J^{T}=-n^{\alpha} n^{\beta} \gamma_{\alpha} \kappa \gamma_{\beta}^{T}
$$

By (8) $\left(\gamma_{\alpha}^{+}=-\gamma_{\alpha}\right)$ we get $\kappa \gamma_{\beta}^{T} \kappa^{-1}=-\gamma_{\beta}$. Thus

$$
J \kappa J^{T}=n^{\alpha} n^{\beta} \gamma_{\alpha} \gamma_{\beta} \kappa .
$$

On the other hand, changing the indices we obtain

$$
J \kappa J^{T}=n^{\beta} n^{\alpha} \gamma_{\beta} \gamma_{\alpha} \kappa .
$$

Thus

$$
2 J \kappa J^{T}=n^{\alpha} n^{\beta}\left[\gamma_{\alpha} \gamma_{\beta}+\gamma_{\beta} \gamma_{\alpha}\right] \kappa=2 n^{\alpha} n^{\beta} \widehat{\eta}_{\alpha \beta} I_{n} \kappa=2 \kappa .
$$

The standard complex structure in the Euclidean space $E_{n}$ is the endomorphism represented by the matrix

$$
J_{0}=\left(\begin{array}{cc}
0 & I_{n / 2} \\
-I_{n / 2} & 0
\end{array}\right) .
$$

It is clear that $J_{0} \in O(n)$.

REMARK 3. For each pair $(k, l)$ of positive integers such that $k+l=n$, we have $J_{0} \notin O(k, l)$.

Pr o of. It suffices to show that $J_{0} \kappa \neq \kappa J_{0}$. Otherwise, we would have $J_{0} \kappa J_{0}^{T}=\kappa J_{0} J_{0}^{T}=\kappa$ and $J_{0}$ would belong to $O(k, l)$.

We divide our proof into 3 parts.

I. $k=l=n / 2$. In this case we have

$$
J_{0} \kappa=\left(\begin{array}{cc}
0 & -I_{n / 2} \\
-I_{n / 2} & 0
\end{array}\right), \quad \kappa J_{0}=\left(\begin{array}{cc}
0 & I_{n / 2} \\
I_{n / 2} & 0
\end{array}\right),
$$

so $J_{0} \kappa \neq \kappa J_{0}$.

II. $k<n / 2$. Then

$$
\begin{aligned}
& J_{0} \kappa=\left(\begin{array}{cc|c}
0 & I_{n / 2} \\
-I_{n / 2} & 0
\end{array}\right)\left(\begin{array}{cc|c}
I_{k} & 0 \\
& -I & \\
\hline 0 & -I_{n / 2}
\end{array}\right)=\left(\begin{array}{cc}
0 & -I_{n / 2} \\
\hline-I_{k} & 0
\end{array}\right), \\
& \kappa J_{0}=\left(\begin{array}{c|cc}
0 & I_{k} & \\
\hline I_{n / 2} & \multicolumn{2}{|c}{0}
\end{array}\right),
\end{aligned}
$$

where $I$ denotes $I_{n / 2-k}$, so in this case $J_{0} \kappa \neq \kappa J_{0}$ as well. 
III. $k>n / 2$. Then

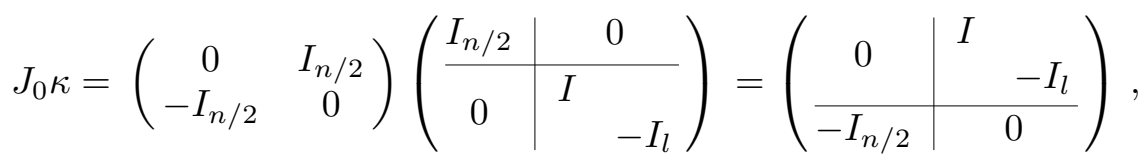

$$
\begin{aligned}
& \kappa J_{0}=\left(\begin{array}{cc|c}
0 & I_{n / 2} \\
\hline-I & & 0
\end{array}\right)
\end{aligned}
$$

where $I$ denotes $I_{n / 2-l}$. Again $J_{0} \kappa \neq \kappa J_{0}$. This completes the proof.

The following problem arises:

Problem 1. For which pairs $(k, l)$ of positive integers does there exist a matrix $J \in O(k, l)$ satisfying $J^{2}=-I_{n}, n=k+l$ ?

We are looking for a matrix $J \in M(n)$ which satisfies

$$
\text { (a) } J^{T} \kappa J=\kappa, \quad \text { (b) } J^{2}=-I_{n} \text {. }
$$

Notice that the above conditions are equivalent to

$$
\text { (a) }(\kappa J)^{T}=-\kappa J, \quad \text { (b) } J^{2}=-I_{n} \text {. }
$$

LEMMA 4. Let

$$
\kappa=\left(\begin{array}{cc}
I_{k} & 0 \\
0 & -I_{l}
\end{array}\right), \quad k, l \neq 0 .
$$

If $B \in O(k, l)$, then

1) $B$ is of the form

$$
B=\left(\begin{array}{cc}
A & C_{1} \\
C_{2} & B
\end{array}\right),
$$

where $A \in M(k), A \neq 0 ; B \in M(l), B \neq 0 ; C_{1} \in M(l \times k), C_{2} \in M(k \times l)$ and the following conditions are satisfied:
(a) $A^{T} A-C_{2}^{T} C_{2}=I_{k}$,
(b) $A^{T} C_{1}-C_{2}^{T} B=0$,
(c) $C_{1}^{T} A-B^{T} C_{2}=0$,
(d) $B^{T} B-C_{1}^{T} C_{1}=I_{l}$.

2) $\operatorname{det} B= \pm 1$.

Proof. The condition 2) is a straightforward consequence of (15). To prove 1) assume that $B$ is of the form (18). Then

$$
B^{T}=\left(\begin{array}{ll}
A^{T} & C_{2}^{T} \\
C_{1}^{T} & B^{T}
\end{array}\right) .
$$

By (15), we have, say,

$$
\left(\begin{array}{cc}
A^{T} & C_{2}^{T} \\
C_{1}^{T} & B^{T}
\end{array}\right)\left(\begin{array}{cc}
I_{k} & 0 \\
0 & -I_{l}
\end{array}\right)\left(\begin{array}{cc}
A & C_{1} \\
C_{2} & B
\end{array}\right)
$$




$$
=\left(\begin{array}{ll}
A^{T} A-C_{2}^{T} C_{2} & A^{T} C_{1}-C_{2}^{T} B \\
C_{1}^{T} A-B^{T} C_{2} & C_{1}^{T} C_{1}-B^{T} B
\end{array}\right)=\left(\begin{array}{cc}
I_{k} & 0 \\
0 & -I_{l}
\end{array}\right) .
$$

This is nothing but (19).

Assume that $A=0$. Then by (19a) we would have $C_{2}^{T} C_{2}=-I_{k}$. If $\left(a_{1}, \ldots, a_{l}\right)$ is the first column of $C_{2}$, then we would get $a_{1}^{2}+\ldots+a_{l}^{2}=-1$, which is impossible. Thus $A \neq 0$. Analogously, we show that $B \neq 0$.

THEOREM 2. Let $\kappa$ be as in Lemma 4. If $J \in O(k, l)$ and $J$ satisfies $J^{2}=-I_{n}, n=k+l$, then

1) $J$ has the form

$$
J=\left(\begin{array}{cc}
A & C \\
C^{T} & B
\end{array}\right)
$$

where $A \in M(k), A \neq 0, A^{T}=-A ; B \in M(l), B \neq 0, B^{T}=-B$; $C \in M(l \times k)$, and the matrices $A, B, C$ satisfy (19) with $C_{1}=C_{2}=C$.

2) The integers $k$ and $l$ are even.

Proof. By the assumptions, $J$ satisfies (17a) so we have

$$
(\kappa J)_{s}^{r}=-(\kappa J)_{r}^{s}, \quad \sum_{m=1}^{n} \kappa_{m}^{r} J_{s}^{m}=-\sum_{w=1}^{n} \kappa_{w}^{s} J_{r}^{w} \quad \text { for } r, s=1, \ldots, n .
$$

Since $\kappa$ is a diagonal matrix, the above equality is equivalent to

$$
\kappa_{r}^{r} J_{s}^{r}=-\kappa_{s}^{s} J_{r}^{s} \quad \text { for } r, s=1, \ldots, n .
$$

By the assumption $\kappa=\operatorname{diag}(\underbrace{1, \ldots, 1}_{k}, \underbrace{-1, \ldots,-1}_{l})$, so by (22) we get the following:

I. If $r \leq k, s \leq k$, then $J_{s}^{r}=-J_{r}^{s}$.

II. If $r>k, s>k$, then $J_{s}^{r}=-J_{r}^{s}$.

III. If $r \leq k, s>k$, then $J_{s}^{r}=J_{r}^{s}$.

IV. If $r>k, s \leq k$, then $J_{s}^{r}=J_{r}^{s}$.

We conclude that $J$ has the form (21). Thus

$$
J^{T}=\left(\begin{array}{cc}
-A & C \\
C^{T} & -B
\end{array}\right) .
$$

Using (17) we get

$$
J^{T} \kappa J=\left(\begin{array}{cc}
-A^{2}-C C^{T} & -A C-C B \\
C^{T} A+B C^{T} & C^{T} C+B^{2}
\end{array}\right)
$$

and

$$
J^{2}=\left(\begin{array}{cc}
A^{2}+C C^{T} & A C+C B \\
C^{T} A+B C^{T} & C^{T} C+B^{2}
\end{array}\right) .
$$


Thus $A, B, C$ satisfy (19) with $C_{1}=C_{2}=C$. Analogously to Lemma 4, we prove that $A, B \neq 0$.

In order to prove the second assertion of our theorem we assume that $k$ and $l$ are odd ( $k+l=n$, and by Lemma $1, n$ is always even). Since $A$ and $B$ are antisymmetric, we then have

$$
\operatorname{det} A=\operatorname{det} B=0 \text {. }
$$

We now show that (23) contradicts (19). Indeed, to the matrix $A^{2}$ we can associate a quadratic form $F_{A^{2}}$ defined by $F_{A^{2}}(x, x):=\left\langle x, A^{2} x\right\rangle$, where $\langle$, denotes the usual scalar product. By (19a) we have

$$
\begin{aligned}
F_{A^{2}}(x, x) & =\left\langle x,\left(-I_{k}-C C^{T}\right) x\right\rangle=\left\langle x,-x-C C^{T} x\right\rangle \\
& =\langle x,-x\rangle-\left\langle x, C C^{T} x\right\rangle=-\|x\|^{2}-\left\langle C^{T} x, C^{T} x\right\rangle \\
& =-\|x\|^{2}-\left\|C^{T} x\right\|^{2}<0
\end{aligned}
$$

for $x \neq 0$. The form $F_{A^{2}}$ is thus negative definite, so $\operatorname{det} A^{2}<0$, which contradicts (23).

REMARK 4. If $k$ and $l$ are even integers $(k+l=n, k, l \neq 0)$, then the matrix $J \in O(k, l)$ satisfying $J^{2}=-I_{n}$ can be chosen as follows:

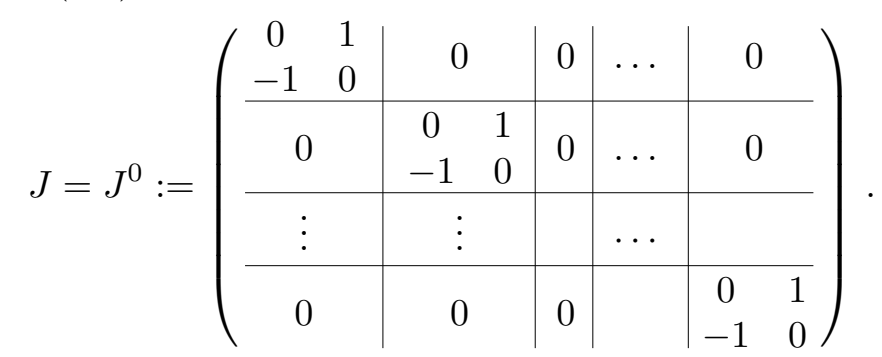

Of course, $\left(J^{0}\right)^{T}=-J^{0}$.

Denote by $F$ the family of all matrices $A \in M(n)$ satisfying one of the equivalent conditions

$$
A^{+}=-A, \quad \kappa A^{T} \kappa^{-1}=-A, \quad(A \kappa)^{T}=-(A \kappa),
$$

where $\kappa^{T}=\kappa=\kappa^{-1}$.

Remark 5. Any $A \in F$ satisfies

$$
\operatorname{Tr} A=0 .
$$

P r o of. Indeed,

$$
(A \kappa)_{j}^{i}=\sum_{m=1}^{n} A_{m}^{i} \kappa_{j}^{m}=A_{j}^{i} \kappa_{j}^{j}
$$

because $\kappa$ is diagonal. Now, since $A \kappa$ is antisymmetric, we get

$$
0=(A \kappa)_{j}^{j}=A_{j}^{j} \kappa_{j}^{j} \Rightarrow A_{j}^{j}=0 .
$$


Corollary 2. The matrices $\gamma_{\alpha}, \alpha=1, \ldots, p, \alpha \neq t$, determined by (7)-(9), belong to $F$.

Corollary 3. If $\gamma_{\alpha}, \alpha=1, \ldots, p, \alpha \neq t$, are the matrices described by $(7)-(9)$ and $\left(n^{\alpha}\right)$ is an arbitrary system of $p-1$ real numbers satisfying $\sum_{\alpha, \beta=1, \alpha, \beta \neq t}^{p} \widehat{\eta}_{\alpha \beta} n^{\alpha} n^{\beta}=1$, then

$$
\operatorname{Tr}\left(i n^{\alpha} \gamma_{\alpha}\right)=0 .
$$

Here the following problem arises:

Problem 2. Determine all matrices $C_{\alpha}, \alpha=1, \ldots, p$, satisfying (5).

Lemma 5. The general formula describing the admissible matrices $C_{\alpha}^{\prime}$ satisfying (5) is

$$
C_{\alpha}^{\prime}=\sum_{\beta} O_{\alpha}^{\beta} R C_{\beta} R^{-1}
$$

where $O \in O(\widehat{\eta}), R \in O(\kappa)$.

Proof. The matrices $C_{\alpha}$ only depend on the choice of the bases in $S$ and $V$. We shall show how the matrices $C_{\alpha}$ transform with the change of the bases. Let

$$
\varepsilon_{\alpha}^{\prime}=O_{\alpha}^{\beta} \varepsilon_{\beta}, \quad e_{j}^{\prime}=R_{j}^{k} e_{k}, \quad R \in O(\kappa), O \in O(\widehat{\eta}),
$$

and

Then

$$
F\left(\varepsilon_{\alpha}^{\prime}, e_{j}^{\prime}\right)=C_{\alpha_{j}}^{\prime k} e_{k}^{\prime}
$$

$$
\begin{aligned}
& F\left(O_{\alpha}^{\beta} \varepsilon_{\beta}, R_{j}^{k} e_{k}\right)=C_{\alpha j}^{\prime k} R_{k}^{m} e_{m}, \\
& O_{\alpha}^{\beta} R_{j}^{k} F\left(\varepsilon_{\beta}, e_{k}\right)=C_{\alpha j}^{\prime k} R_{k}^{m} e_{m}, \\
& O_{\alpha}^{\beta} R_{j}^{k} C_{\beta_{k}}^{l} e_{l}=C_{\alpha j}^{\prime k} R_{k}^{m} e_{m} .
\end{aligned}
$$

Since $R \in O(\kappa)$, it follows that $\kappa R^{T} \kappa^{-1}=R^{-1}, \kappa^{-1}=\kappa$, and

$$
R_{k}^{m}\left(\kappa R^{T} \kappa\right)_{m}^{w}=\delta_{k}^{w}
$$

Thus,

$$
\begin{aligned}
& O_{\alpha}^{\beta} R_{j}^{k} C_{\beta k}^{l} e_{l}=C_{\alpha j}^{\prime k} R_{k}^{m} \delta_{m}^{l} e_{l}, \\
& O_{\alpha}^{\beta} R_{j}^{k} C_{\beta k}^{l}=C_{\alpha j}^{\prime k} R_{k}^{l} .
\end{aligned}
$$

Now, we multiply both sides by $\left(\kappa R^{T} \kappa\right)_{l}^{s}$ :

$$
\begin{aligned}
& O_{\alpha}^{\beta} R_{j}^{k} C_{\beta k}^{l}\left(\kappa R^{T} \kappa\right)_{l}^{s}=C_{\alpha j}^{\prime k} R_{k}^{l}\left(\kappa R^{T} \kappa\right)_{l}^{s}=C_{\alpha j}^{\prime k} \delta_{k}^{s}=C_{\alpha j}^{\prime s}, \\
& O_{\alpha}^{\beta}\left[R C_{\beta} \kappa R^{T} \kappa\right]_{j}^{s}=C_{\alpha j}^{\prime s}, \\
& O_{\alpha}^{\beta} R C_{\beta} R^{-1}=C_{\alpha}^{\prime},
\end{aligned}
$$

as required. It is easy to see that if the matrices $\left(C_{\alpha}\right)$ satisfy (5) then so do the $\left(C_{\alpha}^{\prime}\right)$. 
COROLlaRY 4. The general formula describing the admissible matrices $\gamma_{\alpha}^{\prime}$ satisfying (8) is

$$
\gamma_{\alpha}^{\prime}=O_{\alpha}^{\beta} R \gamma_{\beta} R^{-1},
$$

where $R \in O(\kappa), O \in O(\widehat{\eta})$.

COROLlary 5. If $\left(n^{\alpha}\right)$ is an arbitrary system of numbers satisfying (13) and $\gamma_{\alpha}, \alpha=1, \ldots, p, \alpha \neq t$, is an arbitrary system of matrices determined by (7)-(9) then, changing the base in the space $(V, \kappa)$ by means of an orthogonal transformation $R \in O(\kappa)$, we have the following formula for the admissible matrices $J^{\prime} \in O(k, l)$ satisfying $\left(J^{\prime}\right)^{2}=-I_{n}, n=k+l$ :

$$
J^{\prime}=R J R^{-1},
$$

where $J=i n^{\alpha} \gamma_{\alpha}$.

Now, fix matrices $\gamma_{\alpha}, \alpha=1, \ldots, p, \alpha \neq t$, and a system of $p-1$ real numbers $\left(n^{\alpha}\right)$ satisfying (13). Denote by $\operatorname{Or}\left(J^{0}\right):=\{M \in M(n) ; M=$ $\left.R J^{0} R^{-1}, R \in O(\kappa)\right\}$ the $O(\kappa)$-orbit of the matrix $J^{0}$. Further, let $\operatorname{Or}(J)$ denote the $O(\kappa)$-orbit of $J=i n^{\alpha} \gamma_{\alpha}$. Let us compute the moments of $J^{0}$ and $J$. We have

$$
\begin{aligned}
& \operatorname{Tr} J^{2 k}=\operatorname{Tr}\left(J^{2}\right)^{k}=\operatorname{Tr}\left(-I_{n}\right)^{k}=(-1)^{k} \operatorname{Tr} I_{n}=n(-1)^{k}, \\
& \operatorname{Tr}\left(J^{0}\right)^{2 k}=\operatorname{Tr}\left(J^{02}\right)^{k}=\operatorname{Tr}\left(-I_{n}\right)^{k}=n(-1)^{k}, \quad \text { for } k=1, \ldots, n / 2 .
\end{aligned}
$$

Analogously, by Corollary 3, we have

$$
\operatorname{Tr} J^{2 k+1}=\operatorname{Tr}\left(J^{2 k} \cdot J\right)=\operatorname{Tr}(-J)=0
$$

and, since $J^{0}$ is antisymmetric,

$$
\operatorname{Tr}\left(J^{0}\right)^{2 k+1}=\operatorname{Tr}\left(-J^{0}\right)=0 .
$$

The matrices $J$ and $J^{0}$ have the same moments so they belong to the same orbit of $O(\kappa)$ :

$$
\operatorname{Or}\left(J^{0}\right)=\operatorname{Or}(J) .
$$

Lemma 6. Let $n$ and $p$ be positive integers determined by Lemma 1, $n>1$. Then, to any system $\left(n^{\alpha}\right)$ of $p-1$ real numbers satisfying (13) we can associate a system $\gamma_{\alpha}, \alpha=1, \ldots, p, \alpha \neq t$, of imaginary $n \times n$-matrices satisfying (8) so that

$$
i n^{\alpha} \gamma_{\alpha}=J^{0} .
$$

Proof. By the considerations preceding Lemma 6 , for any system $\left(n^{\alpha}\right)$ of $p-1$ real numbers satisfying (13) and for any system $\gamma_{\alpha}$ of imaginary $n \times n$-matrices satisfying (8) the matrices $J=i n^{\alpha} \gamma_{\alpha}$ and $J^{0}$ belong to the same $O(\kappa)$-orbit. Consequently, by the transitivity of the action of $O(\kappa)$ in this orbit, for each system $\left(n^{\alpha}\right)$ in question there exists an orthogonal transformation of one matrix to the other and so the proof is complete. 
Let us pose the following problem:

Problem 3. Describe the orbit $O(\kappa) \cdot J^{0}$.

Let $\Omega$ and $\Omega^{\prime}$ belong to $O(\kappa) \cdot J^{0}$. Then $\Omega=A J^{0} A^{-1}, \Omega^{\prime}=B J^{0} B^{-1}$, where $A, B \in O(\kappa)$. Notice that

$$
\left(\Omega=\Omega^{\prime}\right) \Leftrightarrow\left[\left(A^{-1} B\right) J^{0}\left(A^{-1} B\right)^{-1}=J^{0}\right] .
$$

Introduce the following relation in $O(\kappa)$ :

$$
(A \sim B) \Leftrightarrow\left[\left(A^{-1} B\right) J^{0}\left(A^{-1} B\right)^{-1}=J^{0}\right] .
$$

It is clear that this is an equivalence relation. Then the set of different matrices $\Omega$ in the orbit $O(\kappa) \cdot J^{0}$ is isomorphic to the group $O(\kappa) / \sim \equiv$ $O(\kappa) / S\left(J^{0}\right)$, where $S\left(J^{0}\right):=\left\{A \in O(\kappa): A J^{0} A^{-1}=J^{0}\right\}$ is the stability group of $J^{0}$.

Let us recall that the endomorphism $\widetilde{n}$ is represented in the basis $\left(e_{j}\right)$ by the matrix

$$
J=i n^{\alpha} \gamma_{\alpha}
$$

where

$$
J=R J^{0} R^{-1}
$$

for some $R \in O(\kappa)$.

Definition. The endomorphism $\widetilde{n}$ described by (4), (8), (12) and (13) will be called a supercomplex structure on $(V, \kappa)$.

This definition is motivated by

LEMMA 7. If a supercomplex structure $\widetilde{n}$ exists, then

$$
\begin{aligned}
& (R e)_{2 j}=J(R e)_{2 j-1}=\widetilde{n}(R e)_{2 j-1}, \\
& (R e)_{2 j-1}=-J(R e)_{2 j}=-\tilde{n}(R e)_{2 j}
\end{aligned}
$$

for some $R \in O(\kappa)$.

Proof. This is a straightforward consequence of Corollaries 4 and 5, Lemma 6, and (11), (13), (30).

Definition. $[(V, \kappa), J, \widetilde{n}, \cdot, \boldsymbol{E}]$ is a complex vector space $[(V, \kappa), J, \cdot]$ equipped with a supercomplex structure $(J, \widetilde{n})$ and a Hurwitz type vector space $\boldsymbol{E}$ of endomorphisms $E: V \rightarrow V$ satisfying

$$
(q+i s) \cdot f=f q+(J f) s \quad \text { for } f \in V \text { and } q, s \in \mathbb{R} .
$$

(By the definition it has to satisfy also the relations (32), (11), (13), and (14).)

TheOREM 3. Consider a pseudo-Euclidean Hurwitz pair $(V(\kappa), S(\eta))$ of bidimension $(n, p), n>1$, and some orthonormal bases $\left(e_{j}\right)$ in $V$ and $\left(\varepsilon_{\alpha}\right)$ in $S$. Let $\left(n^{\alpha}\right)$ be an arbitrary system of real numbers $(13)$ and $\left(\gamma_{\alpha}\right)$ a system 
of imaginary $n \times n$-matrices (8)-(9) with the property (29), which is possible under the assumption that $\kappa=\operatorname{diag}(\underbrace{1, \ldots, 1}_{k=2 k^{\prime}}, \underbrace{-1, \ldots,-1}_{l=2 l^{\prime}}), k^{\prime}, l^{\prime} \neq 0$. Suppose that $f$ is an arbitrary vector in $V$ and let $\sum_{j=1}^{n} e_{j} f_{\mathbb{R}}^{j}$ be its decomposition (in $V)$. Then this decomposition can be rearranged into the form

$$
f=\sum_{j=1}^{n / 2}(R e)_{2 j-1} f^{2 j-1}, \quad \text { where } f^{2 j-1}=E_{0} f_{\mathbb{R}}^{2 j-1}+\widetilde{n} f_{\mathbb{R}}^{2 j}
$$

or

$$
f=\sum_{j=1}^{n / 2}(R e)_{2 j} f^{2 j}, \quad \text { where } f^{2 j}=E_{0} f_{\mathbb{R}}^{2 j}-\widetilde{n} f_{\mathbb{R}}^{2 j-1}
$$

for some $R \in O(\kappa)$, where $\widetilde{n}=\sum_{\alpha=1, \alpha \neq t}^{p} n^{\alpha} E_{\alpha}$.

Proof. The problem whose solution is formulated in Theorem 3 is wellposed by Lemma 1, (11), (13), Theorem 2 and Lemma 6. By (11) and (13),

$$
\widetilde{n} e_{j}=n^{\alpha}\left(i \gamma_{j \alpha}^{k} e_{k}\right)=\left(i n^{\alpha} \gamma_{\alpha}\right)_{j}^{k} e_{k}=J_{j}^{k} e_{k} .
$$

By Lemma $6, \widetilde{n}(R e)_{j}=\left(J^{0}\right)_{j}^{k} e_{k}$. Using Lemma 7, we get

$$
\begin{aligned}
\widetilde{n}(R e)_{2 j-1} & =\left(J^{0}\right)_{2 j-1}^{k}(R e)_{k}=(R e)_{2 j}, \\
\widetilde{n}(R e)_{2 j} & =\left(J^{0}\right)_{2 j}^{k}(R e)_{k}=-(R e)_{2 j-1} .
\end{aligned}
$$

Thus, for every $f=\sum_{j=1}^{n}(R e)_{j} f_{\mathbb{R}}^{j}$ we get

$$
\begin{aligned}
f & =\sum_{j=1}^{n / 2}\left[(R e)_{2 j-1} f_{\mathbb{R}}^{2 j-1}+(R e)_{2 j} f_{\mathbb{R}}^{2 j}\right] \\
& =\sum_{j=1}^{n / 2}\left[(R e)_{2 j-1} f_{\mathbb{R}}^{2 j-1}+\widetilde{n}(R e)_{2 j+1} f_{\mathbb{R}}^{2 j}\right]=\sum_{j=1}^{n / 2}(R e)_{2 j-1} f^{2 j-1},
\end{aligned}
$$

where $f^{2 j-1}:=E_{0} f_{\mathbb{R}}^{2 j-1}+\widetilde{n} f_{\mathbb{R}}^{2 j}$.

Analogously, we obtain (35). The uniqueness of these decompositions is a clear consequence of the uniqueness of $f=\sum_{j=1}^{n} e_{j} f_{\mathbb{R}}^{j}$.

From (34) and (35) we also deduce

LEMMA 8. If $\kappa=\operatorname{diag}(\underbrace{1, \ldots, 1}_{k=2 k^{\prime}}, \underbrace{-1, \ldots,-1}_{l=2 l^{\prime}})$, where $k^{\prime}, l^{\prime} \neq 0$, then by Theorem 3 the decompositions (34) and (35) for $f \in V$ generate the decom- 
positions

$$
V=\bigoplus_{j=1}^{n / 2} C_{j}\left(E_{0}, \widetilde{n}, J\right)
$$

or

$$
V=\bigoplus_{j=1}^{n / 2} \widetilde{C}_{j}\left(E_{0}, \widetilde{n}, J\right),
$$

where $C_{j}\left(E_{0}, \widetilde{n}, J\right)$ and $\widetilde{C}_{j}\left(E_{0}, \widetilde{n}, J\right)$ are complex one-dimensional subspaces of $V$, generated by $e_{2 j-1}$ and $e_{2 j}$, respectively, for $j=1, \ldots, n / 2$. Their dependence on $E_{0}, \widetilde{n}$ and $J$ is determined by (11), (13), and (29).

On the other hand, with the help of the complex structure $J$ we can introduce the complex scalar product $():, V \times V \rightarrow \mathbb{C}$ as follows:

$$
(f, g)=(f, g)_{\mathbb{R}}+i(J f, g)_{\mathbb{R}} \quad \text { for } f, g \in V
$$

(provided $\kappa$, the metric of $V$, satisfies the assumption of Lemma 8), where $(,)_{\mathbb{R}}$ denotes the usual (real) scalar product in $V:(f, g)_{\mathbb{R}}:=\sum_{i=1}^{n} f^{i} g^{i}$ for $f=f^{i} e_{i}, g=g^{i} e_{i}$. Then we have

Proposition 1. The complex scalar product $($,$) has the properties$

$$
\begin{gathered}
(40) \quad(f, g)=\overline{(g, f)}, \quad(f, g+h)=(f, g)+(f, h) \quad \text { for } f, g, h \in V, \\
(41) \quad(f, z g)=z(f, g), \quad(f, f)=\|f\|^{2} \quad \text { for } f, g \in V \text { and } z \in \mathbb{C}, \\
(f, g)=\sum_{j=1}^{n / 2} \overline{f_{\mathbb{C}}^{j}} g_{\mathbb{C}}^{j} \quad \text { for } f, g \in V,
\end{gathered}
$$

where the bar denotes complex conjugation and

$$
\text { (43) } \quad f_{\mathbb{C}}^{j}=f_{\mathbb{R}}^{2 j-1}+i f_{\mathbb{R}}^{2 j}, \quad g_{\mathbb{C}}^{j}=g_{\mathbb{R}}^{2 j-1}+i g_{\mathbb{R}}^{2 j}, \quad j=1, \ldots n / 2
$$

Proof. (40) and (41) follow from (30) and (31) and from the definition of $($,$) and (,)_{\mathbb{R}}$. Indeed,

$$
\begin{aligned}
(g, f) & =(g, f)_{\mathbb{R}}+i(J g, f)_{\mathbb{R}}=(f, g)_{\mathbb{R}}+i(\widetilde{n} g, f)_{\mathbb{R}}=(f, g)_{\mathbb{R}}-n^{\alpha}\left(\gamma_{\alpha} g, f\right) \\
& =(f, g)_{\mathbb{R}}-n^{\alpha} \sum_{k=1}^{n}\left(\gamma_{\alpha} g\right)_{k} f_{k}=(f, g)_{\mathbb{R}}-n^{\alpha} \sum_{k=1}^{n}\left(\sum_{m=1}^{n} \gamma_{\alpha k}^{m} g_{m}\right) f_{k} \\
& =(f, g)_{\mathbb{R}}-n^{\alpha} \sum_{m=1}^{n} \sum_{k=1}^{n} g_{m}\left(-\gamma_{\alpha m}^{k} f_{k}\right) \\
& =(f, g)_{\mathbb{R}}+n^{\alpha} \sum_{m=1}^{n} g_{m}\left(\gamma_{\alpha} f\right)_{m}=(f, g)_{\mathbb{R}}+n^{\alpha}\left(g, \gamma_{\alpha} f\right)_{\mathbb{R}} \\
& =(f, g)_{\mathbb{R}}-i n^{\alpha}\left(g, E_{\alpha} f\right)_{\mathbb{R}}=(f, g)_{\mathbb{R}}-i(g, \widetilde{n} f)_{\mathbb{R}}
\end{aligned}
$$




$$
=(f, g)_{\mathbb{R}}-i(g, J f)_{\mathbb{R}}=(f, g)_{\mathbb{R}}-i(J f, g)_{\mathbb{R}}=\overline{(f, g)} .
$$

In particular,

$$
(f, f)=(f, f)_{\mathbb{R}}+i(J f, f)_{\mathbb{R}}=\overline{(f, f)}=(f, f)_{\mathbb{R}}-i(J f, f)_{\mathbb{R}} .
$$

Hence $(J f, f)_{\mathbb{R}}=0$ and $(f, f)=(f, f)_{\mathbb{R}}=\|f\|^{2}$. The remaining equalities in (40) and (41) are obvious.

To prove (42) we take (36):

$$
\begin{aligned}
(f, g)= & (f, g)_{\mathbb{R}}+i(J f, g)_{\mathbb{R}}=\sum_{k=1}^{n} f^{k} g^{k}+i(\widetilde{n} f, g)_{\mathbb{R}} \\
= & \sum_{k=1}^{n}\left(f^{k} g^{k}+i\left(\widetilde{n}\left(f^{k} e_{k}\right), g\right)_{\mathbb{R}}\right)=\sum_{k=1}^{n} f^{k} g^{k}+i \sum_{j=1}^{n / 2}\left(f^{2 j-1} \widetilde{n}\left(e_{2 j-1}\right)\right. \\
& \left.+f^{2 j} \widetilde{n}\left(e_{2 j}\right), g\right)_{\mathbb{R}}=\sum_{k=1}^{n} f^{k} g^{k}+i \sum_{j=1}^{n / 2}\left(f^{2 j-1} e_{2 j}-f^{2 j} e_{2 j-1}, g\right)_{\mathbb{R}} \\
= & \sum_{k=1}^{n} f^{k} g^{k}+i \sum_{j=1}^{n / 2}\left(f^{2 j-1} g^{2 j}-f^{2 j} g^{2 j-1}\right) \\
= & \sum_{j=1}^{n / 2}\left[f^{2 j-1}\left(g^{2 j-1}+i g^{2 j}\right)+f^{2 j}\left(g^{2 j}-i g^{2 j-1}\right)\right] \\
= & \sum_{j=1}^{n / 2}\left(f^{2 j-1}-i f^{2 j}\right)\left(g^{2 j-1}+i g^{2 j}\right)=\sum_{j=1}^{n / 2} \overline{f_{\mathbb{C}}^{j}} g_{\mathbb{C}}^{j}
\end{aligned}
$$

where $f_{\mathbb{C}}^{j}$ and $g_{\mathbb{C}}^{j}$ are defined by $(43)$.

\section{References}

[1] A. Hurwitz, Über die Komposition der quadratischen Formen von beliebig vielen Variablen, Nachr. Königl. Gesell. Wiss. Göttingen Math.-Phys. Kl. 1898, 308-316; reprinted in: A. Hurwitz, Mathematische Werke II, Birkhäuser, Basel 1933, 565571.

[2] - Über die Komposition der quadratischen Formen, Math. Ann. 88 (1923), 1-25; reprinted in: A. Hurwitz, Mathematische Werke II, Birkhäuser, Basel 1933, 641666 .

[3] J. Ławrynowicz and J. Rembieliński, Hurwitz pairs equipped with complex structures, in: Seminar on Deformations, Łódź - Warsaw 1982/84, Proceedings, J. Ławrynowicz (ed.), Lecture Notes in Math. 1165, Springer, Berlin 1985, 184-197.

[4] -, -, Pseudo-Euclidean Hurwitz pairs and the Katuża-Klein theories, J. Phys. A Math. Gen. 20 (1987), 5831-5848. 
[5] - - - Supercomplex vector spaces and spontaneous symmetry breaking, in: Seminari di Geometria 1984, CNR ed., Università di Bologna, Bologna 1985, 131-154.

INSTITUTE OF MATHEMATICS

POLISH ACADEMY OF SCIENCES

ŁÓDŹ BRANCH

NARUTOWICZA 56

90-136 ŁÓDŹ, POLAND

Reçu par la Rédaction le 5.9.1990 